\title{
Long-term outcomes of dasatinib-induced pulmonary arterial hypertension: a population-based study
}

\author{
Jason Weatherald (10 1,2,3,4 , Marie-Camille Chaumais ${ }^{3,5,6}$, Laurent Savale La $^{1,2,3}$, \\ Xavier Jaïs ${ }^{1,2,3}$, Andrei Seferian ${ }^{1,2,3}$, Matthieu Canuet ${ }^{7}$, Hélène Bouvaist ${ }^{8}$, \\ Pascal Magro ${ }^{9}$, Anne Bergeron ${ }^{10,11}$, Christophe Guignabert (10,3, \\ Olivier Sitbon ${ }^{1,2,3}$, Gérald Simonneau ${ }^{1,2,3}$, Marc Humbert (10 ${ }^{1,2,3}$ and \\ David Montani (1) ${ }^{1,2,3}$
}

\begin{abstract}
Affiliations: 'Université Paris-Sud, Faculté de Médecine, Université Paris-Saclay, Le Kremlin-Bicêtre, France. ${ }^{2}$ Service de Pneumologie, Hôpital Bicêtre, AP-HP, Le Kremlin-Bicêtre, France. ${ }^{3}$ INSERM UMR_S 999, Hôpital Marie Lannelongue, Le Plessis Robinson, France. ${ }^{4}$ Division of Respirology, Dept of Medicine, University of Calgary, Calgary, AB, Canada. ${ }^{5}$ Université Paris-Sud, Faculté de Pharmacie, Université Paris-Saclay, Châtenay Malabry, France. ${ }^{6}$ Service de Pharmacie, DHU-TORINO, Hôpital Antoine Béclère, Clamart, France. ${ }^{7}$ Service de Pneumologie, Nouvel Hôpital Civil, Hôpitaux Universitaires de Strasbourg, Strasbourg. France. ${ }^{8}$ Service de Cardiologie, CHU Genoble Alpes, Grenoble, France. ${ }^{9}$ Service de Pneumologie, CHRU de Tours, Tours, France. ${ }^{10}$ AP-HP, Hôpital Saint-Louis, Service de Pneumologie, Paris, France. ${ }^{11}$ Université Paris-Diderot, Sorbonne Paris Cité, UMR 1153 CRESS, Biostatistics and Clinical Epidemiology Research Team, Paris, France.
\end{abstract}

Correspondence: David Montani, Université Paris-Sud, Centre de Référence de l'Hypertension Pulmonaire, Service de Pneumologie, Hôpital Bicêtre, 78 Rue du général Leclerc, 94270 Le Kremlin-Bicêtre, France. E-mail: david.montaniQaphp.fr

@ERSpublications

Dasatinib-induced pulmonary arterial hypertension persists in over one-third of patients despite stopping dasatinib http://ow.ly/xRPt30csyk9

Cite this article as: Weatherald J, Chaumais M-C, Savale L, et al. Long-term outcomes of dasatinibinduced pulmonary arterial hypertension: a population-based study. Eur Respir J 2017; 50: 1700217 [https://doi.org/10.1183/13993003.00217-2017].

ABSTRACT This study aimed to describe the long-term outcomes of pulmonary arterial hypertension (PAH) induced by dasatinib.

21 incident, right heart catheterisation-confirmed cases of dasatinib-induced PAH were identified from the French Pulmonary Hypertension Registry. Clinical and haemodynamic variables were compared from baseline to last follow-up (median (range) 24 (1-81) months).

Median age was 52 years and 15 patients were female (71\%). 19 patients received dasatinib for chronic myelogenous leukaemia for a median (range) duration of $42(8-74)$ months before PAH diagnosis. No bone morphogenic protein receptor-2 (BMPR2) mutations were found in the 10 patients tested. Dasatinib was uniformly discontinued and 11 patients received PAH medications. Four patients died during followup. New York Heart Association functional class improved from $76 \%$ in class III/IV to $90 \%$ in class I/II $(\mathrm{p}<0.01)$. Median (range) 6-min walk distance improved from $306(0-660)$ to $430(165-635) \mathrm{m}(\mathrm{p}<0.01)$. Median (range) mean pulmonary arterial pressure improved from $45(30-70)$ to $26(17-50) \mathrm{mmHg}$ $(\mathrm{p}<0.01)$ and pulmonary vascular resistance from $6.1(3.2-27.3)$ to $2.6(1.2-5.9)$ Wood units $(\mathrm{p}<0.01)$. Patients treated with $\mathrm{PAH}$ medications had worse baseline haemodynamics but similar long-term outcomes to untreated patients. PAH persisted in $37 \%$ of patients.

Dasatinib-induced PAH frequently improves after discontinuation but persisted in over one-third of patients, therefore systematic follow-up is essential. 


\section{Introduction}

Pulmonary arterial hypertension (PAH) is a severe, progressive disease of the pre-capillary pulmonary arteries, and is defined by an elevation in mean pulmonary arterial pressure (mPAP) $\geqslant 25 \mathrm{mmHg}$ at rest with a normal pulmonary artery wedge pressure (PAWP) $\leqslant 15 \mathrm{mmHg}$ and elevated pulmonary vascular resistance (PVR) $>3$ Wood units (WU) [1]. PAH can be idiopathic, or develop as a result of heritable genetic mutations, exposure to drugs or toxins, connective tissue diseases, congenital heart diseases, portal hypertension and HIV or schistosomiasis infection [2]. All of these causes of PAH share similar pathological features and pathophysiological characteristics, including endothelial cell dysfunction, vasoconstriction, inflammation, in situ thrombosis, proliferation and vascular remodelling [3]. Although significant recent advances have resulted in effective targeted therapies and improved outcomes for patients with PAH, it remains a severe, progressive and ultimately fatal disease in most cases [1].

Dasatinib (Sprycel; Bristol-Myers-Squibb, Princeton, NJ, USA) is an oral second-generation tyrosine kinase inhibitor (TKI) currently approved as a first- or second-line treatment for chronic myelogenous leukaemia (CML) that results in superior short- and long-term response rates compared with imatinib [4, 5]. Dasatinib is also approved for second-line treatment of Philadelphia chromosome-positive acute lymphoblastic leukaemia (ALL) [6]. The family of TKIs targeting BCR-ABL in CML includes imatinib, dasatinib, nilotinib, bosutinib and ponatinib. Compared with imatinib, dasatinib exhibits more potent inhibition of BCR-ABL but also more extensive inhibition of other tyrosine kinases and members of different protein kinase families. These different inhibition profiles may in part explain differences in side-effects, especially the development of PAH and pleural or pericardial effusions in dasatinib-treated patients [7]. Cases of PAH have been predominantly reported in association with dasatinib [8], but also recently with bosutinib $[9,10]$ and ponatinib [11]. Despite being more frequently used than dasatinib, no definite cases of $\mathrm{PAH}$ have been established in association with imatinib or nilotinib, likely due to their higher receptor tyrosine kinase specificity and minimal overlap with dasatinib for other types of protein kinases $[12,13]$. Among the drugs and toxins that can cause PAH, dasatinib is unique in that PAH usually improves or resolves following discontinuation of the offending medication [8]. Although rare, $\mathrm{PAH}$ is now a recognised complication of dasatinib that appears as a warning on the product label [6] with an estimated incidence of at least $0.45 \%$ in chronically treated patients [8].

A recent report sponsored by the manufacturer of dasatinib summarised 41 cases of dasatinib-induced PAH from an international pharmacovigilance database between June 2006 and October 2013, and noted improvement or resolution of PAH in $94 \%$ of cases, with complete resolution in 58\% [14]. However, long-term follow-up was limited in that study and repeat haemodynamic assessment by right heart catheterisation (RHC), the gold standard to diagnose and follow $\mathrm{PAH}$, was performed for only five patients and follow-up echocardiography was done for seven patients. Therefore, the frequency of complete resolution of dasatinib-induced PAH and the long-term outcome of these patients, based on haemodynamic criteria, is unclear.

\section{Material and methods}

Study design and subjects

This was a retrospective study of all incident cases of PAH from the French Pulmonary Hypertension Registry that were confirmed by RHC and associated with dasatinib treatment. The French Pulmonary Hypertension Registry was established in accordance with French bioethics laws (Commission Nationale de l'Informatique et des Libertés) and all patients gave informed consent. All of the identified cases were reported to the French pharmacovigilance network (VIGIAPATH). The main objective was to describe the long-term clinical, functional and haemodynamic outcomes of dasatinib-induced PAH patients from the French Pulmonary Hypertension Registry.

\section{Methods}

Routine evaluation at baseline included medical history, physical examination, echocardiography, contrast-enhanced computed tomography of the chest, ventilation/perfusion lung scan, abdominal ultrasound, autoimmunity screening and HIV serology. Age at diagnosis and clinical status as assessed by

This article has supplementary material available from erj.ersjournals.com

Received: Jan 302017 | Accepted after revision: May 232017

Support statement: J. Weatherald is the recipient of a joint European Respiratory Society/Canadian Thoracic Society Long-Term Research Fellowship (LTRF 2015-4780). Funding information for this article has been deposited with the Crossref Funder Registry.

Conflict of interest: Disclosures can be found alongside this article at erj.ersjournals.com 
modified New York Heart Association (NYHA) functional class and a nonencouraged 6-min walk distance (6MWD) were recorded. Right atrial pressure, mPAP, PAWP and mixed venous oxygen saturation were recorded. Cardiac output was measured by the thermodilution technique and cardiac index was calculated as cardiac output/body surface area. Pulmonary vascular resistance (PVR) was calculated as (mPAP -PAWP)/cardiac output (expressed in WU).

\section{Analysis}

Continuous variables are reported as median (minimum-maximum range). Changes in NYHA functional class, 6MWD and haemodynamics were compared using a paired t-test or the Wilcoxon signed-rank test depending on normality of data using SPSS version 17.0 (SPSS, Chicago, IL, USA). Survival time was calculated from the date of first RHC to the date of last follow-up or death and was analysed using the Kaplan-Meier method. Two patients were switched to bosutinib after stopping dasatinib and experienced worsening PAH on bosutinib [8]. For these two patients, the most recent clinical and haemodynamic follow-up data were used: 6 months after stopping bosutinib for one patient and while on bosutinib treatment for 5 months for the other patient. Graphics were created using Prism version 5.0 (GraphPad, La Jolla, CA, USA).

\section{Results}

Characteristics of patients with dasatinib-induced PAH

From the approval of dasatinib (November 2006) to September 2016, 21 incident patients with dasatinib-induced PAH were identified in the French Pulmonary Hypertension Registry. Short-term outcomes for 11 of these patients have been previously published [8,9] and 10 cases have not been previously reported. Baseline characteristics of 21 subjects are shown in table 1.15 (71\%) patients were female and the median (range) age was $52(17-74)$ years. All patients were receiving dasatinib at the time of diagnosis and the median (range) delay between the initiation of dasatinib and diagnosis of PAH was 42 (8-74) months. 19 patients (90\%) were receiving dasatinib for CML, one patient for ALL and one patient for systemic mastocytosis. Most of the patients (86\%) had received another TKI, mainly imatinib, before initiation of dasatinib. 17 patients received hydroxycarbamide and 10 patients had previously received interferon (IFN)- $\alpha 2 \mathrm{~A}$ or $-\alpha 2 \mathrm{~B}$ in their chemotherapeutic regimens. Bone morphogenic protein receptor-2 (BMPR2) mutation testing was performed in 10 patients, which were all negative. At the time of PAH diagnosis, the dose of dasatinib ranged from 50 to $140 \mathrm{mg} \cdot \mathrm{day}^{-1}$ (median $100 \mathrm{mg} \cdot \mathrm{day}^{-1}$ ). All patients presented with marked clinical and functional impairment: 16 (76\%) patients were NYHA functional class III/IV and median (range) 6MWD was 306 (0-660) m. Isolated pre-capillary pulmonary hypertension was confirmed by RHC in 19 patients, and two patients had combined pre- and post-capillary pulmonary hypertension with elevations in the PVR of 9.2 and $6.4 \mathrm{WU}$ and elevated diastolic pressure gradients of 25 and $18 \mathrm{mmHg}$, respectively. Median (range) mPAP was 45 (3070) $\mathrm{mmHg}$, cardiac index was $3.3(1.4-4.8) \mathrm{L} \cdot \mathrm{min}^{-1} \cdot \mathrm{m}^{-2}$ and PVR was $6.1(3.2-27.3)$ WU. Two patients (9.5\%) met criteria for acute vasoreactivity to nitric oxide [1]. The individual haemodynamic characteristics at diagnosis for all 21 patients are presented in supplementary table S1. Contrast-enhanced computed tomography of the chest and echocardiography showed pleural effusions in 13 patients (62\%) and pericardial effusion in six patients (29\%).

\section{Clinical, functional and haemodynamic evolution after withdrawal of dasatinib}

Dasatinib was discontinued in all patients following confirmation of the diagnosis of PAH. Nine patients received specific $\mathrm{PAH}$ therapy, two patients received calcium channel blockers and treatment with specific PAH therapy was not deemed necessary for 10 patients. After a median (range) clinical follow-up time of 24 (1-81) months, 19 patients had improved at least one NYHA functional class (figure 1), while two patients remained unchanged in NYHA functional class II. At the last evaluation, $90 \%$ of patients were in NYHA functional class I/II (57\% class I, 33\% class II and 10\% class III). A follow-up 6MWD was available for 19 patients (figure 1) and showed significant improvement to a median (range) of $430(165-635) \mathrm{m}$ $(\mathrm{p}<0.01)$. Haemodynamic follow-up (median (range) interval $12(3-81)$ months) was available for 19 patients, which showed improvements (figure 2) in median (range) mPAP from 45 (30-70) to $26(17-50) \mathrm{mmHg}(\mathrm{p}<0.001)$ and PVR from $6.1(3.2-27.3)$ to $2.6(1.2-5.9) \mathrm{WU}(\mathrm{p}<0.001)$. The evolution of haemodynamic and 6MWD improvements for each individual is shown in supplementary figure S1. Improvements in 6MWD occurred within 4 months for most patients. 12 (63\%) out of the 19 patients with haemodynamic follow-up had achieved a normal PVR $(<3 \mathrm{WU})$ within a median (range) time interval of 9 (3-40) months; however, seven patients (37\%) had persistent PAH. Persistent PAH at short-term follow-up RHC (within 4 months of diagnosis) did not predict long-term persistent PAH, as four out of seven patients with PVR $>3 \mathrm{WU}$ at short-term follow-up RHC eventually normalised PVR. Patients who received PAH-specific therapies or calcium channel blockers $(n=11)$ were generally more severe than patients who received no therapy (supplementary table S2). The median cardiac index was significantly lower in patients who received treatment compared with those who did not receive treatment 


\begin{tabular}{|c|c|}
\hline Subjects & 21 \\
\hline Age years & $52(17-74)$ \\
\hline Female & $15(71)$ \\
\hline \multicolumn{2}{|l|}{ Diagnosis } \\
\hline $\mathrm{CML}$ & $19(90)$ \\
\hline ALL & $1(5)$ \\
\hline Systemic mastocytosis & $1(5)$ \\
\hline \multicolumn{2}{|l|}{ TKI treatment prior to dasatinib } \\
\hline Imatinib & $17(81)$ \\
\hline Nilotinib & $2(10)$ \\
\hline None & 4 (19) \\
\hline \multicolumn{2}{|l|}{ Other oncological treatment } \\
\hline Hydroxycarbamide & $17(81)$ \\
\hline Interferon & $10(48)$ \\
\hline Cytarabine & $5(24)$ \\
\hline None & 2 (5) \\
\hline Delay from dasatinib initiation to PAH diagnosis months & $42(8-74)$ \\
\hline Dasatinib dose at PAH diagnosis $\mathrm{mg} \cdot \mathrm{day}^{-1}$ & $100(50-140)$ \\
\hline NYHA FC I/II/III/IV & $0 / 5 / 10 / 6(0 / 24 / 48 / 29)$ \\
\hline Pleural effusions & $13(62)$ \\
\hline Pericardial effusion & $6(29)$ \\
\hline 6MWD m & $306(0-660)$ \\
\hline BNP $n g \cdot L^{-1}$ & $163(49-1635)$ \\
\hline $\mathrm{PaO}_{2} \mathrm{kPa}$ & $9.9(6.3-14)$ \\
\hline Haemoglobin $\mathrm{g} \cdot \mathrm{dL}^{-1}$ & $13.2(7.9-19)$ \\
\hline DLco $\%$ pred & $63(41-98)$ \\
\hline RAP mmHg & $6(3-24)$ \\
\hline mPAP $\mathrm{mmHg}$ & $45(30-70)$ \\
\hline PAWP mmHg & $10(3-23)$ \\
\hline Cardiac output L-min ${ }^{-1}$ & $6.0(2.3-9.2)$ \\
\hline Cardiac index $L \cdot \min ^{-1} \cdot \mathrm{m}^{-2}$ & $3.3(1.4-4.8)$ \\
\hline PVR WU & $6.1(3.2-27.3)$ \\
\hline $\mathrm{SvO}_{2} \%$ & $65(44-85)$ \\
\hline
\end{tabular}

Data are presented as $\mathrm{n}$, median (range) or $\mathrm{n}(\%)$. CML: chronic myelogenous leukaemia; ALL: acute lymphoblastic leukaemia; TKI: tyrosine kinase inhibitor; NYHA FC: New York Heart Association functional class; 6MWD: 6-min walk distance; BNP: brain natriuretic peptide; $\mathrm{PaO}_{2}$ : arterial oxygen tension; $D\llcorner c 0$ : diffusion capacity of the lung for carbon monoxide; RAP: right atrial pressure; mPAP: mean pulmonary artery pressure; PAWP: pulmonary artery wedge pressure; PVR: pulmonary vascular resistance; WU: Wood units; $\mathrm{S}_{2}$ : mixed venous oxygen saturation.
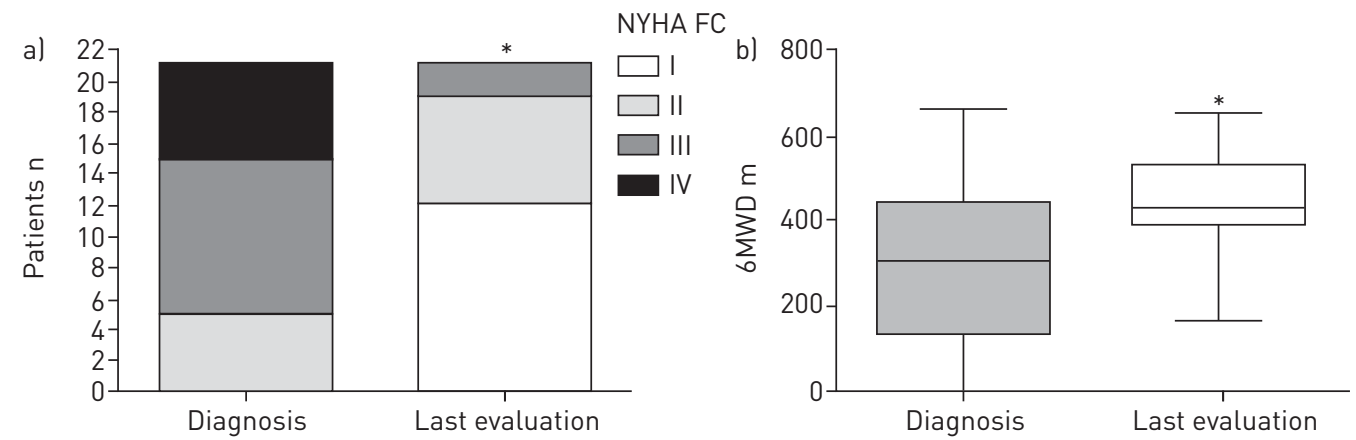

FIGURE 1 Functional changes from diagnosis to last evaluation: a) New York Heart Association functional class (NYHA FC) and b) 6-min walk distance (6MWD). The median (range) duration between diagnosis and the most recent evaluation was 20 (1-81) months for NYHA FC and 18 (3-48) months for 6MWD. There was a significant decrease in the proportion of patients in NYHA FC III/IV to NYHA FC I/II and the mean improvement in 6MWD was $+146 \mathrm{~m}$. ${ }^{*}: p<0.01$. Boxes reflect median with interquartile range and whiskers represent minimum-maximum range. 

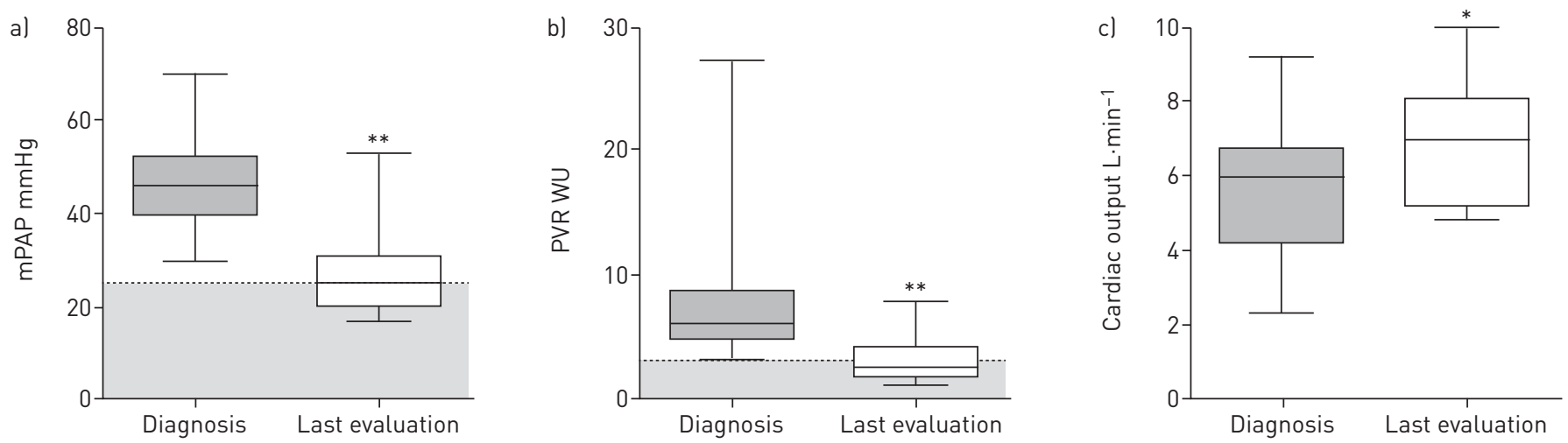

FIGURE 2 Haemodynamic changes from diagnosis to last evaluation. Haemodynamic reassessment was available for 19 patients with a median (range) duration between initial diagnostic right heart catheterisation (RHC) to last RHC of 12 (3-81) months. Changes from baseline to most recent $\mathrm{RHC}$ in a) mean pulmonary arterial pressure (mPAP), which decreased from a median (range) of 45 (30-70) to 26 (17-50) mmHg, b) pulmonary vascular resistance (PVR), which decreased from a median (range) of 6.1 (3.2-27.3) to 2.6 (1.2-5.9) Wood units (WU) and c) cardiac output, which increased from a median (range) of $6.0(2.3-9.2)$ to $6.9(4.9-9.9) \mathrm{L} \cdot \mathrm{min}^{-1}$. $^{*}: \mathrm{p}=0.03$; **: $\mathrm{p}<0.001$. The shaded areas below the dashed lines reflect an absence of pulmonary arterial hypertension (PAH) if a) mPAP $<25 \mathrm{mmHg}$ and b) PVR $<3 \mathrm{WU}$. Seven patients (37\%) had persistent $\mathrm{PAH}$ at last follow-up, defined as mPAP $\geqslant 25 \mathrm{mmHg}$, pulmonary artery wedge pressure $\leqslant 15 \mathrm{mmHg}$ and PVR $>3$ WU. Boxes reflect median with interquartile range and whiskers represent minimum-maximum range.
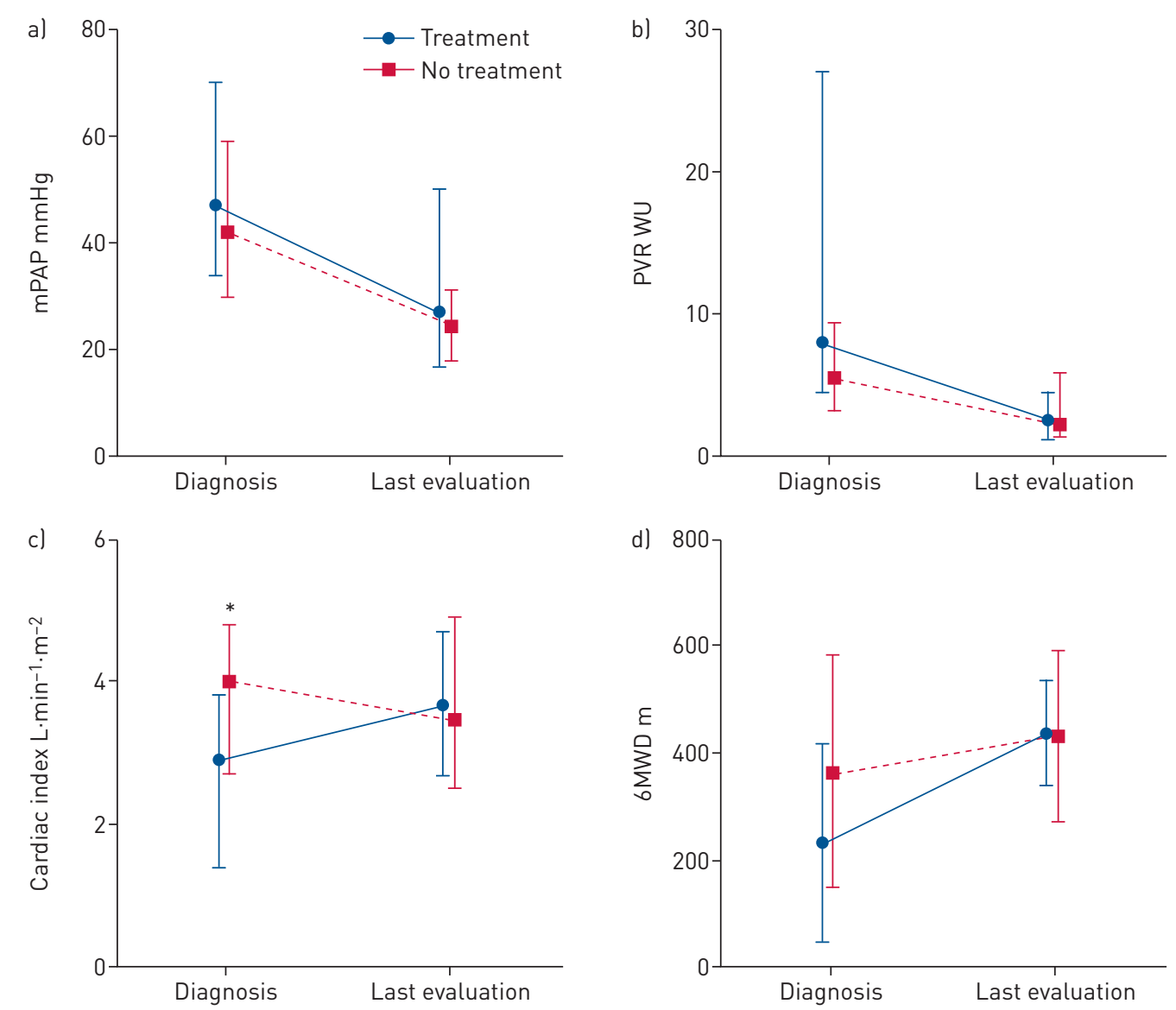

FIGURE 3 Changes in haemodynamic variables and 6-min walk distance (6MWD) by treatment strategy. Changes in haemodynamics $(n=19)$ from baseline to last evaluation according to initial treatment strategy after the discontinuation of dasatinib: no treatment $(n=8)$, treatment group that received pulmonary arterial hypertension (PAH)-specific treatment $(n=9)$ and calcium channel blocker therapy $(n=2)$. Median (range) values for a) mean pulmonary artery pressure (mPAP) and b) pulmonary vascular resistance (PVR) were not significantly different between treatment groups at baseline or last evaluation, although baseline PVR was higher in the group who received $\mathrm{PAH}$-specific treatment compared with those who received no treatment $(8$ versus 5.8 Wood units (WU); $p=0.14$ ). c) Baseline median cardiac index was significantly lower in the treatment group.*: $p<0.01$. d) Baseline median 6MWD was nonsignificantly lower in the treatment group ( $p=0.15$ ). 
FIGURE 4 Kaplan-Meier survival function for 21 patients with dasatinib-induced pulmonary arterial hypertension. $90.5 \%, 90.5 \%$ and $85.7 \%$ of patients were still alive at 1,3 and 5 years, respectively.

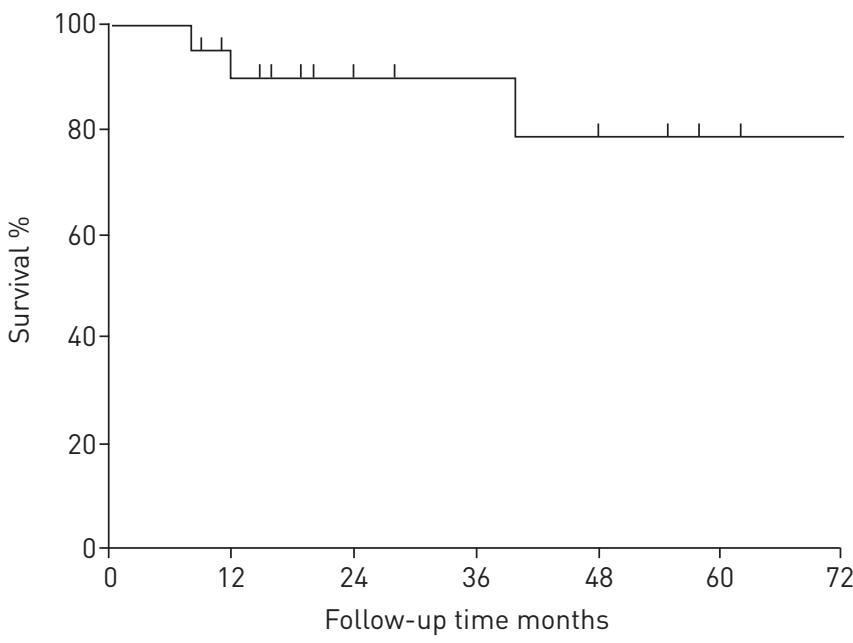

At risk $\mathrm{n}$

21
18

11

9

4

2

(figure 3). Changes in 6MWD and haemodynamics according to treatment group for each individual patient are shown in supplementary figure S2. Among the 12 patients who normalised haemodynamics, three had exercise haemodynamic testing and two of these patients had pulmonary hypertension during exercise, defined as peak $\mathrm{mPAP}>30 \mathrm{mmHg}$ and total pulmonary resistance $>3 \mathrm{WU}$ [15], at 36 and 9 months follow-up, respectively. One patient with persistent $\mathrm{PAH}$, who had initially improved with $\mathrm{PAH}$ therapy, developed haemodynamic worsening 81 months after diagnosis, following a therapeutic de-escalation from a combination of a phosphodiesterase type 5 (PDE-5) inhibitor and endothelin receptor antagonist ("ERA to ERA") monotherapy. Two patients experienced worsening PAH after switching to bosutinib [9]. Four patients died at 8, 12, 39 and 74 months after pulmonary hypertension diagnosis, resulting in 1-, 3- and 5-year survival rates of $90.5 \%, 90.5 \%$ and $85.7 \%$, respectively (figure 4 ). One patient died of heart failure in the context of Candida albicans septicaemia, one died suddenly a few days after a long-distance flight [8] and no information was available on the cause of death for the other two patients.

Based on the experience from patients in the current study and other published cases $[10,11,14,16-19]$, we propose a management algorithm for dasatinib-induced PAH to help guide clinical decisions (figure 5). Given the small number of reported cases of dasatinib-induced $\mathrm{PAH}$, this algorithm reflects current guidelines [1] for PAH diagnosis and treatment in addition to incorporating our opinions on the optimal clinical management of dasatinib-induced PAH and should not be considered an official recommendation.

\section{Discussion}

This is the first study to report long-term clinical and haemodynamic outcomes in a cohort of patients with dasatinib-induced $\mathrm{PAH}$ and, to the best of our knowledge, is the largest series of dasatinib-induced $\mathrm{PAH}$. A novel finding of this study is that, while the majority of patients improved after discontinuation of dasatinib, more than one-third had persistent PAH when reassessed by RHC during long-term follow-up. Furthermore, $43 \%$ remained symptomatic with NYHA functional class II/III symptoms despite discontinuation of dasatinib. This underscores the importance of long-term surveillance of all patients who develop PAH associated with dasatinib, regardless of whether initial improvements occur after discontinuation. We also report characteristics and outcomes according to whether specific treatment was administered, which may help guide future management of patients with dasatinib-induced PAH.

Our finding that PAH persisted long-term in seven out of 19 patients (37\%) despite discontinuation of dasatinib suggests that dasatinib can cause irreversible pulmonary vascular dysfunction and remodelling, which is supported by experimental animal models [20]. One patient in our study experienced haemodynamic worsening after withdrawal of a PAH-specific medication 81 months after diagnosis, and two patients remained symptomatic and had exercise pulmonary hypertension despite normal resting haemodynamic measures, lending further support to the hypothesis that dasatinib can also induce irreversible pulmonary vascular disease. In line with our results, a recent study by SHAH et al. [14] also reported that complete resolution of PAH occurred in 58\% of patients; however, the majority of these cases had no documented follow-up RHC or echocardiography. We have more definitively demonstrated with systematic follow-up RHC that the majority of patients with dasatinib-induced PAH do normalise haemodynamics over the long-term. 


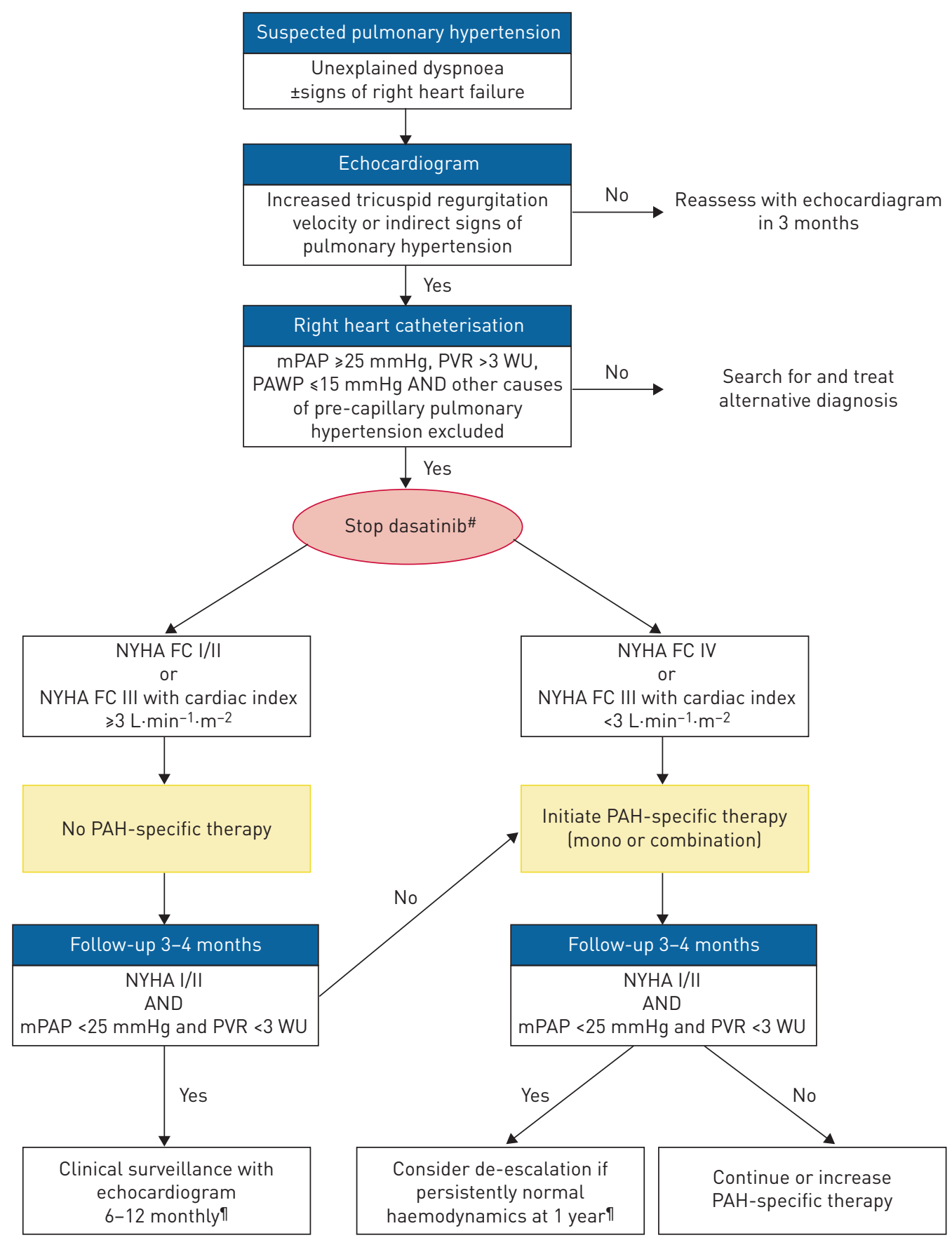

FIGURE 5 Proposed management algorithm for dasatinib-induced pulmonary arterial hypertension (PAH). mPAP: mean pulmonary arterial pressure; PVR: pulmonary vascular resistance; PAWP: pulmonary artery wedge pressure; WU: Wood units; NYHA FC: New York Heart Association functional class. " : a multidisciplinary discussion is recommended to decide which tyrosine kinase inhibitor (TKI) should be initiated in place of dasatinib; ๆ : more frequent follow-up evaluations or continuation of PAH-specific treatment may be warranted if bosutinib or ponatinib are initiated due to the potential risk of recurrent/worsening PAH with these TKIs.

From our analysis of long-term clinical and haemodynamic outcomes, it is difficult to conclude whether $\mathrm{PAH}$-specific therapy is effective or unnecessary in dasatinib-induced PAH. Although we observed similar clinical and haemodynamic outcomes in treated and nontreated patients at last follow-up (figure 3), patients who received treatment with $\mathrm{PAH}$-specific medications (i.e. endothelin receptor antagonists or PDE-5 inhibitors) tended to be more severe at baseline. Specifically, more of the treated patients had presented in NYHA functional class IV and they had a significantly lower cardiac index at diagnosis. Furthermore, a higher proportion of the treated patients had persistent PAH at last follow-up RHC despite treatment, suggesting their more severe clinical presentation was associated with irreversible pulmonary 
vascular remodelling. Therefore, given these baseline differences between the treatment groups and nonrandomised treatment decisions, a beneficial effect of PAH-specific therapy in the most severe patients cannot be excluded.

In our series, four patients (19\%) died at 8, 12, 39 and 74 months after PAH diagnosis, respectively (figure 4). Only one of these patients had evidence of persistent PAH at the time of death. The $85.7 \%$ survival at 5 years in this study is comparable to the 5-year overall survival rates of $80.6-93.8 \%$ reported for CML patients treated with first-line dasatinib in clinical trials [5], suggesting that PAH contributes to morbidity but may not necessarily contribute to an increased risk of death in most patients if $\mathrm{PAH}$ is properly diagnosed and dasatinib is discontinued. Although rare, at least one death attributable to dasatinib-induced $\mathrm{PAH}$ has been reported in the literature; a recent case report from Japan described a 36-year-old woman with CML who developed dasatinib-induced PAH and died from right heart failure despite discontinuation of dasatinib and treatment with catecholamines and a PDE-5 inhibitor [16]. Therefore, PAH-specific therapy is justified in severe cases that present with poor prognostic factors such as right heart failure, a low cardiac index or in NYHA functional class IV (figure 5). For those patients in NYHA functional class III with a cardiac index $\geqslant 3.0 \mathrm{~L} \cdot \mathrm{min}^{-1} \cdot \mathrm{m}^{-2}$ and for patients presenting in NYHA functional class I/II, discontinuation of dasatinib alone without treatment and with a follow-up RHC within 3-4 months is reasonable. In milder cases, PAH-specific treatment could be reserved for patients with inadequate improvement by 3-4 months or with objective worsening despite discontinuation of dasatinib. Continuation of $\mathrm{PAH}$-specific therapies and/or closer surveillance may be warranted if the introduction of bosutinib or ponatinib is being considered, as there is a risk of worsening or recurrent PAH with these TKIs [9-11].

Additional environmental or genetic factors, a so-called "second hit", are necessary to incite the development of PAH in a susceptible individual $[8,20]$. Our results do lead to several hypotheses regarding the nature of these predisposing factors and secondary insults. None of our patients were found to carry BMPR2 mutations to suggest a genetic predisposition to dasatinib-induced PAH; however, BMPR2 status was not available in 11 patients and other predisposing genetic mutations associated with PAH were not assessed in this study. As there was a female predominance in our series (as opposed to a male majority in CML clinical trials [4]), we can hypothesise that sex-related hormonal, pharmacokinetic or immunological differences could contribute to the risk of dasatinib-induced $\mathrm{PAH}$, although this remains speculative. The long delay (median 42 months) between the initiation of dasatinib and the development of PAH suggests that an adequately long exposure to dasatinib is necessary before a secondary insult can trigger the development of PAH. While other causes of pulmonary hypertension were excluded at the time of diagnosis, many patients had previous exposure to chemotherapeutic drugs (including IFN- $\alpha$, hydroxycarbamide and imatinib) and two patients reported previous brief $(<2$ months) exposure to anorexigen drugs (dexfenfluramine $n=1$ and benfluorex $\mathrm{n}=1$ ). IFN- $\alpha$ has been associated with the development of $\mathrm{PAH}$, most often in the setting of chronic hepatitis C infection [21-23], and both dexfenfluramine and benfluorex are known causes of drug-induced $\mathrm{PAH}$ [1]. Of the seven patients with persistent $\mathrm{PAH}$, one was exposed to IFN- $\alpha$ and one was exposed to dexfenfluramine. Therefore, these medications could interact with dasatinib to increase individual susceptibility to mitochondrial oxidative stress and the development of PAH [20]. For the remaining four patients with persistent $\mathrm{PAH}$, no other risk factors were identified, suggesting that other factors such as individual variation in susceptibility to the effects of oxidative stress, genetic polymorphisms or other as-yet unrecognised environmental factors could have contributed.

Receptor tyrosine kinases, including the receptors for platelet-derived growth factor, c-Kit and Src, among others, are involved in the pathogenesis of PAH [24, 25]. This led to interest in TKIs as potential therapeutic options for PAH patients [26]. However, a randomised trial of imatinib in patients with PAH found no reduction in clinical worsening of PAH and there was a high rate of adverse events [27]. Despite interest in TKIs for treating PAH [26, 27], dasatinib and other TKIs (bosutinib and ponatinib) paradoxically seem to incite the development of $\mathrm{PAH}$, which is likely due to more widespread inhibition of off-target receptor and nonreceptor tyrosine kinases or other types of protein kinases that are not affected by imatinib (figure 6) $[7-13,28]$. As dasatinib more potently inhibits BCR-ABL, induction of CML cellular apoptosis does not require continuous target inhibition, in contrast to imatinib where sensitivity increases with increasing exposure time [29]. Older age ( $>65$ years old) and dasatinib dosing regimens other than $100 \mathrm{mg}$ once daily appear to be associated with higher rates of pleural effusion, suggesting that dose exposure is related to the degree of endothelial dysfunction [5, 30-32]. In pharmacokinetic studies, steady-state trough levels of dasatinib are lowest at the $100 \mathrm{mg}$ once-daily dose and patients taking this dose have the lowest incidence of pleural effusion [30, 31, 33]. The extent of off-target protein kinase inhibition by dasatinib is far wider than imatinib within the normal range of dasatinib plasma concentrations [34]. Thus, more than once-daily dosing of dasatinib can result in unnecessarily higher total exposure to dasatinib, which also prolongs off-target inhibition, increasing the potential for adverse 


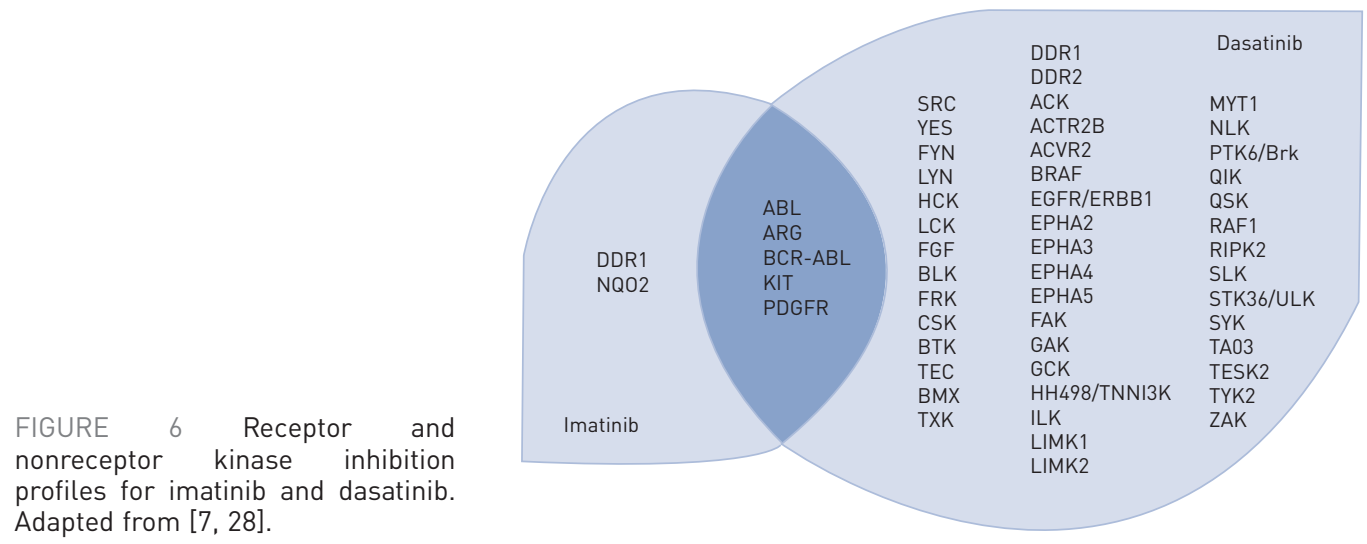

secondary effects $[30,34]$. Although individual variation in pharmacokinetics results in markedly different plasma concentrations between patients for a similar dose, it is not yet established whether individualisation of dasatinib dosing regimens based on trough levels will translate to a decreased incidence of dasatinib-related complications, including PAH [35]. In our current study, steady-state dasatinib plasma concentrations at the time of PAH diagnosis were not assessed and therefore we cannot confirm this hypothesis that pharmacokinetic variability contributes to PAH susceptibility.

The mechanisms by which PAH develops during dasatinib treatment have been partially elucidated in recent studies. Dasatinib also more potently targets the Src family of tyrosine kinases than imatinib [12]. Inhibition of Src by dasatinib results in pulmonary vasoconstriction and increased pulmonary artery pressure through the inhibition of the TWIK-related acid sensitive potassium-1 channel [36]. It had therefore been hypothesised that Src-mediated vasoconstriction, rather than vascular remodelling, might explain the reversible nature of dasatinib-induced PAH in humans $[19,36]$. A recent experimental study in rats and human pulmonary endothelial cells demonstrated that dasatinib causes dose-dependent pulmonary endothelial dysfunction, injury and apoptosis, which is mediated by the production of mitochondrial reactive oxygen species independent of Src inhibition [20]. In this recent study by GUIGNABERT et al. [20], CML patients treated with dasatinib also had higher markers of endothelial injury, such as soluble intercellular adhesion molecule-1, soluble vascular cell adhesion molecule-1 and soluble E-selectin, than treatment-naive patients with CML, patients treated with imatinib and healthy controls. Furthermore, pre-treatment of rats with high doses of dasatinib caused exaggerated pulmonary arterial remodelling in response to a second insult such as hypoxia or monocrotaline. This is consistent with our finding of a high rate of persistent pulmonary vascular disease, as seven patients had persistent PAH and two additional patients had exercise pulmonary hypertension.

We acknowledge several limitations in the current study given its retrospective nature and that short-term outcomes for some of these cases have previously been reported. Most of the treated patients have remained on therapy and therefore it is not clear if PAH may have evolved favourably without treatment or would have progressed in these patients without treatment. As a result, our management algorithm in figure 5 is not based on strong evidence. Furthermore, the optimal timing of PAH medication withdrawal in patients who improve is not well defined based on our results. A final limitation is the possibility that management and outcomes of the 12 patients diagnosed after publication of the first nine cases in France [8] were influenced by improved awareness of this complication among haematologists and earlier diagnosis. A greater proportion of patients diagnosed after 2012 received PAH-specific treatment (eight out of 12) compared with three out of the initial nine cases, perhaps due to greater experience and knowledge that complete resolution of dasatinib-induced PAH was not universal [8]. However, the 12 patients diagnosed after 2012 also had slightly worse cardiac index at diagnosis (median cardiac index 3.2 versus $3.6 \mathrm{~L} \cdot \mathrm{min}^{-1} \cdot \mathrm{m}^{-2}$ ), which could also have influenced management decisions.

In conclusion, we report the long-term clinical and haemodynamic outcomes for 21 patients with dasatinib-induced $\mathrm{PAH}$, which was persistent in $37 \%$ of patients. Although four patients died during follow-up, no deaths were directly attributable to PAH and long-term survival was comparable to that of TKI-treated CML patients without PAH. With persistent symptoms and haemodynamic abnormalities in a significant proportion of patients, and the potential for relapse when specific $\mathrm{PAH}$ medications are discontinued, ongoing surveillance is necessary by physicians involved in the care of these patients. Management of dasatinib-induced $\mathrm{PAH}$ necessitates permanent discontinuation and PAH medications may be helpful in severe cases. 


\section{Acknowledgements}

We acknowledge the French pulmonary hypertension pharmacovigilance network (VIGIAPATH), supported by the Agence Nationale de Sécurité du Médicament et des Produits de Santé (ANSM). We thank the patients, their families and healthcare providers from the French Pulmonary Hypertension Network for agreeing to collaborate. We acknowledge the support of the European Respiratory Society (ERS) and the Canadian Thoracic Society (CTS), joint ERS/CTS Fellowship LTRF 2015.

\section{References}

1 Galie N, Humbert M, Vachiéry J-L, et al. 2015 ESC/ERS Guidelines for the diagnosis and treatment of pulmonary hypertension: The Joint Task Force for the Diagnosis and Treatment of Pulmonary Hypertension of the European Society of Cardiology (ESC) and the European Respiratory Society (ERS): Endorsed by: Association for European Paediatric and Congenital Cardiology (AEPC), International Society for Heart and Lung Transplantation (ISHLT). Eur Respir J 2015; 46: 903-975.

2 Simonneau G, Gatzoulis MA, Adatia I, et al. Updated clinical classification of pulmonary hypertension. J Am Coll Cardiol 2013; 62: D34-D41.

3 Guignabert C, Tu L, Girerd B, et al. New molecular targets of pulmonary vascular remodeling in pulmonary arterial hypertension. Chest 2015; 147: 529-537.

4 Kantarjian H, Shah NP, Hochhaus A, et al. Dasatinib versus imatinib in newly diagnosed chronic-phase chronic myeloid leukemia. N Engl J Med 2010; 362: 2260-2270.

5 Cortes JE, Saglio G, Kantarjian HM, et al. Final 5-year study results of DASISION: the Dasatinib Versus Imatinib Study in Treatment-Naïve Chronic Myeloid Leukemia Patients trial. J Clin Oncol 2016; 34: 2333-2340.

6 US Food and Drug Administration. Sprycel (dasatinib) prescribing and labeling information. www.accessdata.fda gov/drugsatfda_docs/label/2010/021986s7s8lbl.pdf Date last accessed January 10, 2017.

7 Hantschel O, Rix U, Superti-Furga G. Target spectrum of the BCR-ABL inhibitors imatinib, nilotinib and dasatinib. Leuk Lymphoma 2008; 49: 615-619.

8 Montani D, Bergot E, Günther S, et al. Pulmonary arterial hypertension in patients treated by dasatinib. Circulation 2012; 125: 2128-2137.

9 Riou M, Seferian A, Savale L, et al. Deterioration of pulmonary hypertension and pleural effusion with bosutinib following dasatinib lung toxicity. Eur Respir J 2016; 48: 1517-1519.

10 Hickey PM, Thompson AA, Charalampopoulos A, et al. Bosutinib therapy resulting in deterioration of pre-existing pulmonary arterial hypertension. Eur Respir J 2016; 48: 1514-1516.

11 Quilot F-M, Georges M, Favrolt N, et al. Pulmonary hypertension associated with ponatinib therapy. Eur Respir J 2016; 47: 676-679

12 Rix U, Hantschel O, Dürnberger G, et al. Chemical proteomic profiles of the BCR-ABL inhibitors imatinib, nilotinib, and dasatinib reveal novel kinase and nonkinase targets. Blood 2007; 110: 4055-4063.

13 Winter GE, Rix U, Carlson SM, et al. Systems-pharmacology dissection of a drug synergy in imatinib-resistant CML. Nat Chem Biol 2012; 8: 905-912.

14 Shah NP, Wallis N, Farber HW, et al. Clinical features of pulmonary arterial hypertension in patients receiving dasatinib. Am J Hematol 2015; 90: 1060-1064.

15 Herve P, Lau EM, Sitbon O, et al. Criteria for diagnosis of exercise pulmonary hypertension. Eur Respir J 2015; 46: 728-737.

16 Morishita S, Hagihara M, Itabashi M, et al. Development of pulmonary arterial hypertension during oral dasatinib therapy for chronic myelogenous leukemia. Rinsho Ketsueki 2016; 57: 999-1003.

17 Rasheed W, Flaim B, Seymour JF. Reversible severe pulmonary hypertension secondary to dasatinib in a patient with chronic myeloid leukemia. Leuk Res 2009; 33: 861-864.

18 Mattei D, Feola M, Orzan F, et al. Reversible dasatinib-induced pulmonary arterial hypertension and right ventricle failure in a previously allografted CML patient. Bone Marrow Transplant 2009; 43: 967-968.

19 Groeneveldt JA, Gans SJM, Bogaard HJ, et al. Dasatinib-induced pulmonary arterial hypertension unresponsive to PDE-5 inhibition. Eur Respir J 2013; 42: 869-870.

20 Guignabert C, Phan C, Seferian A, et al. Dasatinib induces lung vascular toxicity and predisposes to pulmonary hypertension. J Clin Invest 2016; 126: 3207-3218.

21 Savale L, Sattler C, Günther S, et al. Pulmonary arterial hypertension in patients treated with interferon. Eur Respir J 2014; 44: 1627-1634.

22 Savale L, Chaumais MC, Sitbon O, et al. Pulmonary arterial hypertension in patients treated with interferon. Eur Respir J 2015; 46: 1851-1853.

23 Savale L, Chaumais MC, O'Connell C, et al. Interferon-induced pulmonary hypertension: an update. Curr Opin Pulm Med 2016; 22: 415-420.

24 Perros F, Montani D, Dorfmüller P, et al. Platelet-derived growth factor expression and function in idiopathic pulmonary arterial hypertension. Am J Respir Crit Care Med 2008; 178: 81-88.

25 Montani D, Perros F, Gambaryan N, et al. C-kit-positive cells accumulate in remodeled vessels of idiopathic pulmonary arterial hypertension. Am J Respir Crit Care Med 2011; 184: 116-123.

26 Ghofrani H, Morrell N, Hoeper M, et al. Imatinib in pulmonary arterial hypertension patients with inadequate response to established therapy. Am J Respir Crit Care Med 2010; 182: 1171-1177.

27 Hoeper MM, Barst RJ, Bourge RC, et al. Imatinib mesylate as add-on therapy for pulmonary arterial hypertension: results of the randomized IMPRES study. Circulation 2013; 127: 1128-1138.

28 Swords R, Mahalingam D, Padmanabhan S, et al. Nilotinib: optimal therapy for patients with chronic myeloid leukemia and resistance or intolerance to imatinib. Drug Des Devel Ther 2009; 3: 89-101.

29 Snead JL, O'Hare T, Adrian LT, et al. Acute dasatinib exposure commits Bcr-Abl-dependent cells to apoptosis. Blood 2009; 114: 3459-3463.

30 Wang X, Roy A, Hochhaus A, et al. Differential effects of dosing regimen on the safety and efficacy of dasatinib: retrospective exposure-response analysis of a Phase III study. Clin Pharmacol 2013; 5: 85-97.

31 Shah NP, Kantariian HM, Kim D-W, et al. Intermittent target inhibition with dasatinib $100 \mathrm{mg}$ once daily preserves efficacy and improves tolerability in imatinib-resistant and -intolerant chronic-phase chronic myeloid leukemia. J Clin Oncol 2008; 26: 3204-3212. 
32 Jutant E-M, Phan C, Tu L, et al. Dasatinib causes pulmonary endothelial damage and increases endothelial permeability. Am J Respir Crit Care Med 2016; 193: A2797.

33 Porkka K, Khoury HJ, Paquette RL, et al. Dasatinib $100 \mathrm{mg}$ once daily minimizes the occurrence of pleural effusion in patients with chronic myeloid leukemia in chronic phase and efficacy is unaffected in patients who develop pleural effusion. Cancer 2010; 116: 377-386.

34 Kitagawa D, Yokota K, Gouda M, et al. Activity-based kinase profiling of approved tyrosine kinase inhibitors. Genes Cells 2013; 18: 110-122.

35 Josephs DH, Fisher DS, Spicer J, et al. Clinical pharmacokinetics of tyrosine kinase inhibitors: implications for therapeutic drug monitoring. Ther Drug Monit 2013; 35: 562-587.

36 Nagaraj C, Tang B, Balint Z, et al. Src tyrosine kinase is crucial for potassium channel function in human pulmonary arteries. Eur Respir J 2013; 41: 85-95. 